\title{
THE MAXIMAL IDEAL SPACE OF FUNCTIONS LOCALLY APPROXIMABLE IN A FUNCTION ALGEBRA $^{1}$
}

\author{
C. E. RICKART
}

Introduction. Let $\Omega$ be a compact Hausdorff space and $C(\Omega)$ the Banach algebra of all complex-valued continuous functions on $\Omega$. Also let $\mathfrak{R}$ be a closed subalgebra of $C(\Omega)$ which separates the points of $\Omega$ and contains the constants. We shall also assume that $\Omega$ is the "carrier space" or "space of maximal ideals" of $\mathfrak{A}$. This amounts to requiring that every homomorphism $\phi$ of $\mathfrak{A}$ on to the complex numbers be given by a point of $\Omega[5, \S 3.1]$. In other words, there is a point $\omega_{\phi}$ such that $\hat{a}(\phi)=a\left(\omega_{\phi}\right)$ for every $a \in \mathfrak{A}$, where $\hat{a}(\phi)$ denotes the image of $a$ under the homomorphism $\phi$. Obviously every point of $\Omega$ determines such a homomorphism.

A complex function $f$ defined in $\Omega$ is said to be locally approximable in $\mathfrak{A}$ if every point of $\Omega$ has a neighborhood in which $f$ is a uniform limit of elements of $\mathfrak{A}$. We also call such functions $\mathfrak{R}$-holomorphic [4]. If every point of $\Omega$ has a neighborhood in which $f$ actually coincides with an element of $\mathfrak{P}$. then $f$ is said to belong locally to $\mathfrak{A}$. The $\mathfrak{A}$ holomorphic functions are obviously continuous and contain the functions that belong locally to $\mathfrak{H}$. The closure in $C(\Omega)$ of the $\mathfrak{P}$ holomorphic functions will be denoted by $\mathfrak{B}_{1}$ and the closure of the functions that belong locally to $\mathfrak{A}$ will be denoted by $\mathfrak{B}_{0}$. Evidently $\mathfrak{A} \subseteq \mathfrak{B}_{0} \subseteq \mathfrak{B}_{1} \subseteq C(\Omega)$, and both $\mathfrak{B}_{3}$ and $\mathfrak{B}_{1}$ are subalgebras of $C(\Omega)$.

As is shown by an example of Eva Kallin's [2], it may happen that $\mathfrak{B}_{0} \neq \mathfrak{l}$. On the other hand, G. Stolzenberg [7] has proved that $\mathfrak{B}_{0}$ nevertheless must have $\Omega$ as its space of maximal ideals. The object of this paper is to prove that the same conclusion holds for the algebra $\mathfrak{B}_{1}$. In fact we shall prove the more inclusive result that $\Omega$ is the space of maximal ideals for any closed subalgebra $\mathfrak{B}$ of $C(\Omega)$ which contains $\mathfrak{A}$ and is generated by $\mathfrak{A}$-holomorphic functions. In particular, the Stolzenberg result is included. Stolzenberg's proof depends on the nontrivial fact that $\mathfrak{i}$ satisfies a local maximum modulus principle $[6,6.1$, p. 9]. Our proof, though quite different in spirit, also rests ultimately on the local maximum principle since it involves a result concerning convexity of abstract analytic varieties which we have obtained using this principle [4]. The theorem ob-

Received by the editors March 10, 1966.

1 The research for this paper was partially supported by NSF Grant GP-5493. 
tained here is actually a special case of more general results in the theory of $\mathfrak{A}$-holomorphic functions which we expect eventually to publish elsewhere.

In $\$ 1$ we obtain some elementary properties of the algebra of polynomials in an infinite number of variables regarded as functions on an infinite dimensional complex space. The main theorem is proved in $\$ 2$. In $\$ 3$ we give an example to show that the $\mathfrak{A}$-holomorphic functions need not form a closed subset of $C(\Omega)$.

1. Polynomials in an infinite number of variables. Let $\Lambda$ be an arbitrary set of indices and associate with each $\lambda \in \Lambda$ a complex plane $\boldsymbol{C}_{\lambda}$. Denote by $\boldsymbol{C}^{\boldsymbol{\Lambda}}$ the cartesian product of all the planes $\boldsymbol{C}_{\boldsymbol{\lambda}}$. Let $\Omega$ be the algebra of all polynomials in the complex variables $\left\{\zeta_{\lambda}: \lambda \in \Lambda\right\}$ which have complex coefficients. Thus each $P \in \Omega$ is of the form

$$
P\left(\left\{\zeta_{\lambda}\right\}\right)=\sum_{k_{1}+\cdots+k_{n} \leqq N} \alpha_{k_{1}} \cdots k_{n} \zeta_{\lambda_{1}}^{k_{1}} \cdots \zeta_{\lambda_{n}}^{k_{n}},
$$

where $\lambda_{1}, \cdots, \lambda_{n}$ is any finite subset of $\Lambda$, the exponents $k_{1}, \cdots, k_{n}$ are nonnegative integers, the coefficients $\alpha_{k_{1}} \cdots k_{n}$ are complex numbers, and $N$ is a fixed nonnegative integer depending on $P$. The algebra operations in $\Omega$ are the usual ones for polynomials. We may therefore regard $\Omega$ as an algebra of functions on $C^{\Lambda}$. Note that this is an algebra of continuous functions with the usual product space topology for $\boldsymbol{C}^{\Lambda}$.

The algebra $\Re$ has the elementary but important property that all of its homomorphisms on to the complex numbers are given by points of $\boldsymbol{C}^{\Lambda}\left[\mathbf{5}\right.$, p. 149]. To see this, let $Z_{\mu}$ denote, for each $\mu \in \Lambda$, the polynomial $Z_{\mu}\left(\left\{\zeta_{\lambda}\right\}\right)=\zeta_{\mu}$. Then each $P \in \Omega$ is of the form

$$
P=\sum_{k_{1}+\cdots+k_{n} \leqq N} \alpha_{k_{1}} \cdots k_{n} Z_{\lambda_{1}}^{k_{1}} \cdots Z_{\lambda_{n}}^{k_{n}}
$$

and therefore a homomorphism $\phi$ of $\Omega$ on to the complex numbers has the form

$$
\hat{P}(\phi)=\sum \alpha_{k_{1}} \cdots k_{n} \hat{Z}_{\lambda_{1}}(\phi)^{k_{1}} \cdots \hat{Z}_{\lambda_{n}}(\phi)^{k_{n}}=P\left(\left\{\hat{Z}_{\lambda}(\phi)\right\}\right)
$$

for each $P \in \Omega$. Thus $\phi$ is given by the point $\left\{\hat{Z}_{\lambda}(\phi)\right\}$ of $C^{\Delta}$.

Consider next the cartesian product $\Omega \times C^{\Lambda}$ and let $\mathfrak{A} \times \Omega$ denote the ordinary Kronecker product of the two algebras $\mathfrak{A}$ and $\Omega$. Then $\mathfrak{Q} \times \Omega$ may be identified with the algebra of all functions on $\Omega \times C^{\wedge}$ of the form 


$$
F\left(\omega,\left\{\zeta_{\lambda}\right\}\right)=\sum_{k=1}^{n} a_{k}(\omega) P_{k}\left(\left\{\zeta_{\lambda}\right\}\right),
$$

where $a_{k} \in \mathfrak{A}$ and $P_{k} \in \mathfrak{R}$. Thus $\mathfrak{A} \times \mathfrak{R}$ may be regarded as a subalgebra of $C\left(\Omega \times \mathbf{C}^{\boldsymbol{\Delta}}\right)$.

1.1. Lemma. Every homomorphism of $\mathfrak{A} \times \mathfrak{\Omega}$ onto the complex numbers is given by a point of $\Omega \times C^{\Lambda}$.

Proof. For each $a \in \mathfrak{R}$, define

$$
F_{a}\left(\omega,\left\{\zeta_{\lambda}\right\}\right)=a(\omega), \quad\left(\omega,\left\{\zeta_{\lambda}\right\}\right) \in \Omega \times C^{\Lambda} .
$$

Then the mapping $a \rightarrow F_{a}$ is an isomorphism of $\mathfrak{A}$ in to $\mathfrak{A} \times \Re$. Similarly, if we define

$$
F_{P}\left(\omega,\left\{\zeta_{\lambda}\right\}\right)=P\left(\left\{\zeta_{\lambda}\right\}\right), \quad\left(\omega,\left\{\zeta_{\lambda}\right\}\right) \in \Omega \times C^{\Lambda},
$$

for each $P \in \Re$, then $P \rightarrow F_{P}$ is an isomorphism of $\Re$ into $\mathfrak{A} \times \Re$. Now if $\phi$ is a homomorphism of $\mathfrak{A} \times \mathfrak{\Omega}$ on to the complex numbers, then the mappings

$$
a \rightarrow \hat{F}_{a}(\phi) \text { and } P \rightarrow \hat{F}_{P}(\phi)
$$

define homomorphisms of $\mathfrak{A}$ and $\Omega$ respectively onto the complex numbers. Hence there exist $\omega_{\phi} \in \Omega$ and $\left\{\delta_{\lambda}\right\} \in C^{\Lambda}$ such that

$$
\hat{F}_{a}(\phi)=a\left(\omega_{\phi}\right) \text { and } \hat{F}_{P}(\phi)=P\left(\left\{\delta_{\lambda}\right\}\right)
$$

for all $a \in \mathfrak{Y}$ and $P \in \mathfrak{\Omega}$. Moreover, since each $F$ in $\mathfrak{A} \times \mathfrak{\Omega}$ is of the form

$$
F=\sum_{k=1}^{n} F_{a_{k}} F_{P_{k}}, \quad a_{k} \in \mathfrak{A}, \quad P_{k} \in \Re,
$$

we have

$$
\begin{aligned}
\hat{F}(\phi) & =\sum_{k=1}^{n} \hat{F}_{a_{k}}(\phi) \hat{F}_{P_{k}}(\phi)=\sum_{k=1}^{n} a_{k}\left(\omega_{\phi}\right) P_{k}\left(\left\{\delta_{\lambda}\right\}\right) \\
& =F\left(\omega_{\phi},\left\{\delta_{\lambda}\right\}\right),
\end{aligned}
$$

which completes the proof of the lemma.

2. The maximal ideal space of $\mathfrak{B}$. Recall that $\mathfrak{B}$ is any closed subalgebra of $C(\Omega)$ which contains $\mathfrak{A}$ and has a system of generators $\left\{z_{\lambda}: \lambda \in \Lambda\right\}$ consisting of $\mathfrak{A}$-holomorphic functions. The elements $\left\{z_{\lambda}\right\}$, which may be infinite in number, are generators in the sense that the smallest subalgebra of $\mathfrak{B}$ which contains $\left\{z_{\lambda}\right\}$ is dense in $\mathfrak{B}$. Consider the polydisc 


$$
\Delta=\left\{\left\{\zeta_{\lambda}\right\}:\left|\zeta_{\lambda}\right| \leqq\left|z_{\lambda}\right|_{\Omega}=\sup _{\omega}\left|z_{\lambda}(\omega)\right|, \lambda \in \Lambda\right\},
$$

in $C^{\Lambda}$. It is obvious that $\Delta$ is compact and is $\Omega$-convex (i.e. $\Delta$ consists of all points $\left\{\zeta_{\lambda}\right\} \in C^{\Lambda}$ such that $\left|P\left(\left\{\zeta_{\lambda}\right\}\right)\right| \leqq|P|_{\Delta}$, for every $P \in \Omega$, where $|P|_{\Delta}$ is the maximum absolute value of $P$ on $\left.\Delta\right)$. It is also readily verified that $\Omega \times \Delta$ is a compact $\mathfrak{A} \times \Omega$-convex subset of $\Omega \times C^{\Lambda}$. Now for each $\omega \in \Omega$ define $\tilde{\omega}=\left(\omega,\left\{z_{\lambda}(\omega)\right\}\right)$. Then $\omega \rightarrow \tilde{\omega}$ is the generalized "Oka mapping" [3, p. 15] of $\Omega$ in to $\Omega \times \Delta$. We denote the image of $\Omega$ in $\Omega \times \Delta$ by $\tilde{\Omega}$.

Next we define for each $\mu \in \Lambda$ the function

$$
H_{\mu}\left(\omega,\left\{\zeta_{\lambda}\right\}\right)=z_{\mu}(\omega)-\zeta_{\mu}, \quad\left(\omega,\left\{\zeta_{\lambda}\right\}\right) \in \Omega \times \mathbf{C}^{\Lambda} .
$$

Since $z_{\mu}$ is $\mathfrak{A}$-holomorphic in $\Omega$, it follows immediately that $H_{\mu}$ is $\mathfrak{A} \times \mathfrak{\Omega}$-holomorphic in $\Omega \times C^{\Lambda}$. Furthermore, we have

$$
\tilde{\Omega}=\left\{\left(\omega,\left\{\zeta_{\lambda}\right\}\right): H_{\mu}\left(\omega,\left\{\zeta_{\lambda}\right\}\right)=0, \mu \in \Lambda\right\} .
$$

Thus $\tilde{\Omega}$ is an $\mathfrak{A} \times \Re$-analytic subvariety of the compact $\mathfrak{A} \times \Re$-convex set $\Omega \times \Delta$ according to the definition of this notion in $[4, \S 3]$. By Lemma 1.1, $\mathfrak{U} \times \Re$ is a "natural" algebra of functions on $\Omega \times C^{\Delta}$ in the sense of [4]. Therefore, by [4, Theorem 3.2], it follows that $\tilde{\Omega}$ is also an $\mathfrak{A} \times \mathfrak{\Omega}$-convex set. In other words, $\tilde{\Omega}$ consists of all points $\left(\omega,\left\{\zeta_{\lambda}\right\}\right)$ in $\Omega \times C^{\Lambda}$ such that $\left|F\left(\omega,\left\{\zeta_{\lambda}\right\}\right)\right| \leqq|F|_{\tilde{\Omega}}$, for every $F$ in $\mathfrak{A} \times \Omega$. The way is now clear to prove the main theorem.

2.1. Theorem. The space of maximal ideals of the Banach algebra $\mathfrak{B}$ is equal to $\Omega$.

Proof. Let

$$
F=\sum_{k=1}^{n} a_{k} P_{k}, \quad a_{k} \in \mathfrak{A}, \quad P_{k} \in \Re,
$$

be an arbitrary element of $\mathfrak{U} \times \Re$. Since the polynomials $P_{k}$ involve only a finite number of the variables $\zeta_{\lambda}$ and since $\mathfrak{B}$ is an algebra containing $\mathfrak{A}$, it is meaningful to write

$$
b_{F}=\sum_{k=1}^{n} a_{k} P_{k}\left(\left\{z_{\lambda}\right\}\right),
$$

and $b_{F}$ is an element of $\mathfrak{B}$. Observe that $b_{F}(\omega)=F(\tilde{\omega})$ for all $\omega \in \Omega$. Hence the mapping $F \rightarrow b_{F}$ is a homomorphism of the algebra $\mathfrak{A} \times \Re$ into $\mathfrak{B}$. Moreover, if we define

$$
F_{\mu}\left(\omega,\left\{\zeta_{\lambda}\right\}\right)=\zeta_{\mu}, \quad\left(\omega,\left\{\zeta_{\lambda}\right\}\right) \in \Omega \times \boldsymbol{C}^{\Delta}
$$


then $F_{\mu} \in \mathfrak{A} \times \Re$ and $b_{F_{\mu}}=z_{\mu}$ for each $\mu \in \Lambda$. Thus the image of $\mathfrak{A} \times \Re$ in $\mathfrak{B}$ contains the generators and is therefore a dense subalgebra of $\mathfrak{B}$.

Now let $\zeta$ be any homomorphism of $\mathfrak{B}$ onto the complex numbers. Then the mapping $F \rightarrow \hat{b}_{F}(\phi)$ defines a similar homomorphism of $\mathfrak{U} \times \Omega$. Hence by Lemma 1.1 there exists a point $\left(\omega_{\phi},\left\{\delta_{\lambda}\right\}\right)$ in $\Omega \times C^{\Delta}$ such that

$$
F\left(\omega_{\phi},\left\{\delta_{\lambda}\right\}\right)=\hat{b}_{F}(\phi), \quad F \in \mathfrak{U} \times \mathfrak{B} .
$$

Furthermore, since $\mathfrak{B}$ is a Banach algebra, we always have $|\hat{b}(\phi)|$ $\leqq|b|_{\Omega}$, so

$$
\left|\hat{b}_{F}(\phi)\right| \leqq \sup _{\omega}\left|b_{F}(\omega)\right|=\sup _{\tilde{\omega}}|F(\tilde{\omega})|=|F| \tilde{\Omega},
$$

Thus

$$
\left|F\left(\omega_{\phi},\left\{\delta_{\lambda}\right\}\right)\right| \leqq|F|_{\Omega}, \quad F \in \mathfrak{U} \times \Re .
$$

Since $\tilde{\Omega}$ is $\mathfrak{A} \times \Omega$-convex, it follows that $\left(\omega_{\phi},\left\{\delta_{\lambda}\right\}\right) \in \tilde{\Omega}$. Therefore $\tilde{\omega}_{\phi}=\left(\omega_{\phi},\left\{\delta_{\lambda}\right\}\right)$ and hence

$$
\hat{b}_{F}(\phi)=F\left(\tilde{\omega}_{\phi}\right)=b_{F}\left(\omega_{\phi}\right), \quad F \in \mathfrak{A} \times \mathfrak{B} .
$$

Finally, since elements of the form $b_{F}$ are dense in $\mathfrak{B}$ and $\phi$ is continuous, we must have $\hat{b}(\phi)=b\left(\omega_{\phi}\right)$ for every $b \in \mathfrak{B}$. In other words, every homomorphism of $\mathfrak{B}$ onto the complex numbers is given by a point of $\Omega$ which implies that $\Omega$ is the maximal ideal space of $\mathfrak{B}$.

2.2. REMARK. If the Šilov boundary $\partial \mathscr{q} \Omega$ of $\Omega$ with respect to $\mathfrak{A}$ is a proper subset of $\Omega$, then there always exist closed subalgebras of $C(\Omega)$ which contain $\mathfrak{A}$ and have spaces of maximal ideals larger than $\Omega$. For example let $\subseteq$ be the algebra of all functions in $C(\Omega)$ whose restrictions to $\partial x \Omega$ coincide with an element of $\mathfrak{A}$. If for $c \in \mathbb{C}$ we denote by $a_{c}$ the element of $\mathfrak{A}$ which coincides with $c$ on $\partial x \Omega$ and let $\sigma$ be any point of $\Omega$ not in $\partial थ \Omega$, then it is readily verified that the mapping $c \rightarrow a_{c}(\sigma)$ defines a homomorphism of $\sqrt{5}$ onto the complex numbers which is not given by (evaluation at) a point of $\Omega$. Actually, all such "extra" homomorphisms of 5 are obtained in this way. However in this case $\partial_{\S} \Omega=\Omega$ and, in particular, $\partial_{\S} \Omega \neq \partial_{\mathfrak{A}} \Omega$, while, for $\mathfrak{B}_{1}$, we have $\partial_{\mathfrak{B}_{1}} \Omega=\partial_{\mathscr{r}} \Omega[1$, p. $925 ; 4,2.4]$. Nevertheless, as is shown by an example due to D. R. Wilken [8], the condition that the Silov boundaries be the same is still not sufficient to ensure that $\Omega$ be the space of maximal ideals of the larger algebra. It may suffice to assume that the Šilov boundaries be the same for every $\mathfrak{A}$-convex subset of $\Omega$. Such a condition may be thought of as a requirement that the larger algebra satisfy the same local maximum modulus principle as $\mathfrak{A}$. It is possible that a result of this kind is accessible through methods 
similar to those used here, although they do not seem to apply in their present form.

3. An example. We present here an example which shows that the $\mathfrak{A}$-holomorphic functions need not be closed in $C(\Omega)$. It is based on Eva Kallin's example [2] of an algebra $\Omega$ for which there exist functions that belong locally to the algebra without being elements of it. Recall that the space of maximal ideals for $\Omega$ is a compact set $\Gamma$ in $C^{4}$.

First choose a bounded open polydisc $\Delta$ which contains $\Gamma$. For each $n$ let $\tau_{n}$ be a homeomorphism of $\Delta$ onto a polydisc $\Delta_{n}$ such that $\left\{\Delta_{n}\right\}$ is a disjoint sequence which converges to a single point $\sigma_{x}$. Set $\Gamma_{n}=\tau_{n}(\Gamma)$ and, for each $f$ in $C(\Gamma)$, define

$$
f^{\tau_{n}}\left(\tau_{n}(\sigma)\right)=f(\sigma), \quad \sigma \in \Gamma .
$$

Then the mapping $f \rightarrow f^{\tau_{n}}$ is a norm-preserving isomorphism of $C(\Gamma)$ onto $C\left(\Gamma_{n}\right)$. Let $\Omega_{n}$ denote the image of $\Omega$ in $C\left(\Gamma_{n}\right)$ under this isomorphism. Then $\Re_{n}$ is a closed subalgebra of $C\left(\Gamma_{n}\right)$ with $\Gamma_{n}$ as its space of maximal ideals. Next define $\Omega$ to be the union of all of the sets $\Gamma_{n}$ plus the point $\sigma_{\infty}$. Then $\Omega$ is a compact set in $C^{4}$. Denote by $\mathfrak{A}$ the family of all $f$ in $C(\Omega)$ such that the restriction of $f$ to the set $\Gamma_{n}$ belongs to $\Omega_{n}$. Then $\mathfrak{A}$ is a closed subalgebra of $C(\Omega)$ and has $\Omega$ for its space of maximal ideals [5, 3.2.21].

Now let $u$ be a function that belongs locally to $\Omega$ but does not belong to $\Omega$ and define the function $g$ as follows:

$$
g(\omega)= \begin{cases}2^{-n} u^{\tau_{n}}(\omega), & \omega \in \Gamma_{n}, \\ 0, & \omega=\sigma_{\infty} .\end{cases}
$$

Then $g \in C(\Omega)$. Also, for each $n$, define

$$
g_{n}(\omega)= \begin{cases}g(\omega), & \omega \in \bigcup_{k=1}^{n} \Gamma_{k}, \\ 0, & \omega \in \Omega-\bigcup_{k=1}^{n} \Gamma_{k} .\end{cases}
$$

Then $\lim g_{n}=g$ in $C(\Omega)$. Moreover, each $g_{n}$ belongs locally to $\mathfrak{A}$ and is hence $\mathfrak{A}$-holomorphic. However, since each neighborhood of $\sigma_{\infty}$ contains sets $\Gamma_{n}$ for sufficiently large $n$, it follows that $g$ is not locally approximable in $\mathfrak{A}$ at the point $\sigma_{\infty}$. Therefore the $\mathfrak{A}$-holomorphic functions are not closed in $C(\Omega)$.

\section{BIBLIOGRAPHY}

1. I. Glicksberg, Maximal algebras and a theorem of Rado, Pacific J. Math. 14 (1964), 919-941. 
2. Eva Kallin, A non-local function algebra, Proc. Nat. Acad. Sci. U. S. A. 49 (1963), 821-824.

3. K. Oka, Sur les fonctions analytiques de plusieures variables, Iwanami Shoten, Tokyo, 1961.

4. C. E. Rickart, Analytic phenomena in general function algebras, Pacific J. Math. 18 (to appear).

5. - - General theory of Banach algebras, Van Nostrand, Princeton, N. J., 1960.

6. H. Rossi, The local maximum modulus principle, Ann. of Math. 72 (1960), 1-11.

7. G. Stolzenberg The maximal ideal space of the functions locally in a function algebra, Proc. Amer. Math. Soc 14 (1963), 342-345.

8. D. R. Wilkin, Maximal ideal spaces and A-convexity, Proc. Tulane Conf. on Function Algebras, pp. 120-121, Frank T. Birtel, Editor, Scott, Foresman and Co., Chicago, Ill., 1966.

\section{YALE UNIVERSITY}

